\title{
A Spectral Collocation Technique for Riesz Fractional Chen-Lee- Liu Equation
}

\author{
M. A. Abdelkawy $\mathbb{D}^{1,2}$ and S. A. Alyami $\mathbb{D}^{1}$ \\ ${ }^{1}$ Department of Mathematics and Statistics, Faculty of Science, Imam Mohammad Ibn Saud Islamic University (IMSIU), \\ Riyadh, Saudi Arabia \\ ${ }^{2}$ Department of Mathematics, Faculty of Science, Beni-Suef University, Beni Suef, Egypt
}

Correspondence should be addressed to M. A. Abdelkawy; melkawy@yahoo.com

Received 5 January 2021; Revised 1 February 2021; Accepted 24 March 2021; Published 14 April 2021

Academic Editor: Ahmed S. Hendy

Copyright (c) 2021 M. A. Abdelkawy and S. A. Alyami. This is an open access article distributed under the Creative Commons Attribution License, which permits unrestricted use, distribution, and reproduction in any medium, provided the original work is properly cited.

\begin{abstract}
This paper discusses the study of optical solitons that are modeled by Riesz fractional Chen-Lee-Liu model, one of the versions of the famous nonlinear Schrödinger equation. This model is solved by the assistance of consecutive spectral collocation technique with two independent approaches. The first is the approach of the spatial variable, while the other is the approach of the temporal variable. It is concluded that the method of the current paper is far more efficient and credible for the proposed problem. Numerical results illustrate the performance efficiency of the algorithm. The results also point out that the scheme can lead to spectral accuracy of the studied model.
\end{abstract}

\section{Introduction}

Several numerical methods, including local and global methods, have been listed as approximation techniques for treating the differential equations. The local methods listed the approximate solution at specific points, while the global methods give the approximate solution in whole the mentioned interval. The numerical approximations for differential equations [1-4] are listed at specific points using finite difference methods. While the finite element methods subdivide the whole interval into subintervals and give the approximate solution in them. The finite element methods are used for various types of differential equation; see for example [5-7].

Recently, there are more interests of appointing the spectral methods to treat with various kinds of differential and integral equations $[8,9]$, due to their applicability to bounded and unbounded domains $[10,11]$. The convergence speed is one of the major advantages of spectral method. Spectral methods are promising candidates for solving fractional differential equations since their global nature fits well with the nonlocal definition of fractional operators. They have gained new popularity in automatic computations for a wide class of different problems which included linear and nonlinear differential equation of integer or fractional (fixed, variable, Riesz, tempered, and distributed orders); see $[12,13]$. Also, they are more reliable to treat the integral and integro-differential equations. Spectral methods have exponential convergence rates as well as a high accuracy level. The spectral method has been classified into four classes, collocation [14], tau [15], Galerkin [16], and Petrov-Galerkin [17] methods.

The theory of optical solitons [18-21] is mainly governed by the well-known nonlinear Schrödinger equation (NLSE) [22-25]. However, there exists a wide variety of its manifestations and modifications that also govern pulse transfer across the globe through optical fibers, PCF, metamaterials, and couplers. A few such models are Schrödinger-Hirota equation [26], Manakov equation, complex GinzburgLandau equation, Fokas-Lenells equation, Gabitov-Turitsyn equation, and many others. These models are considered under different circumstances such as dispersive solitons, differential group delay, and dispersion-managed solitons. Besides these familiar models, there is another class of 
versions of NLSE that is referred to as derivative NLSE (DNLSE) [27-29] that appears in three forms. One such form is the Chen-Lee-Liu equation [30-32] that incorporates higher order perturbations from optics and is going to be the focus of today's paper. While a plethora of preexisting work has been already reported in regard to this model, today's focus is going to be handling the model by the aid of fully shifted Legendre collocation method.

Shifted Legendre collocation schemes are used to numerically solve the Riesz fractional Chen-Lee-Liu model. The solution $\Theta(\xi, \tau)$ is firstly placed in its real $\mathscr{U}(\xi, \tau)$ and imaginary $\mathscr{V}(\xi, \tau)$ parts. Accordingly, the real $\mathscr{U}(\xi, \tau)$ and imaginary $\mathscr{V}(\xi, \tau)$ parts of such equation are approximated as $\mathcal{U}_{\mathcal{N}, \mathcal{M}}(\xi, \tau)$ and $\mathcal{U}_{\mathcal{N}, \mathcal{M}}(\xi, \tau)$, respectively, which can be expressed as a finite expansion of shifted Legendre polynomials for spatial variable. Subsequently, the Chen-Lee-Liu equation with boundary conditions is reduced to temporal differential system with initial conditions. Then, the shifted Legendre-Gauss-Radau collocation is assigned for temporal discretization, which is more reliable for treating with such problems. Substituting these discretizations in the mentioned equation gets a nonlinear system of algebraic equations which solved numerically using the Newton-Raphson approach.

This paper is arranged as follows. In Section 1, some properties of Riemann-Liouville fractional derivatives, shifted Legendre polynomials, and shifted Chebyshev polynomials are listed. The mentioned scheme is implemented for the Chen-Lee-Liu equation with initial-boundary conditions in Section 2. In Section 3, two test examples are discussed. The competence of our numerical approach is exhibited by diverse examples in Section 4 . Few remarks are mentioned in the last section (Section 5).

\section{Riemann-Liouville Fractional Derivative}

The fractional integration of order $\mu>0$ exists in different formulas [33]. Riemann-Liouville formula, the most common and widely used, is defined as follows:

$$
\begin{gathered}
J^{\mu} f(\zeta)=\frac{1}{\Gamma(\mu)} \int_{0}^{\zeta}(\zeta-\tau)^{\mu-1} f(\tau) d \tau, \quad \mu>0, \zeta>0, \\
J^{0} f(\zeta)=f(\zeta) .
\end{gathered}
$$

Here, we introduce some properties of the fractional operators. The left-sided and the right-sided fractional derivatives of Riemann-Liouville type of order $\beta(n-1<\beta<n)$ are defined as follows:

$$
\begin{aligned}
& { }_{-\infty} D_{\xi}^{\beta} \psi(\xi, \tau)=\frac{1}{\Gamma(n-\beta)} \frac{\partial^{n}}{\partial \xi^{n}} \int_{-\infty}^{\xi}(\xi-z)^{n-1-\beta} \psi(z, \tau) d z, \\
& { }_{\xi} D_{+\infty}^{\beta} \psi(\xi, \tau)=\frac{(-1)^{n}}{\Gamma(n-\beta)} \frac{\partial^{n}}{\partial \xi^{n}} \int_{\xi}^{+\infty}(z-\xi)^{n-1-\beta} \psi(z, \tau) d z
\end{aligned}
$$

The Riesz fractional derivative is defined as follows:

$$
\frac{\partial^{\beta}}{\partial|\xi|^{\beta}} \psi(\xi, \tau)=-(-\nabla)^{\beta / 2} \psi(\xi, \tau)=c_{\beta}\left[-\infty D_{\xi}^{\beta} \psi(\xi, \tau)+{ }_{\xi} D_{+\infty}^{\beta} \psi(\xi, \tau)\right],
$$

where $c_{\beta}=-1 / 2 \cos (\pi \beta / 2)$. The fractional Laplacian operator in Equation (3) can be represented in the following equivalent Fourier form on the spatial variable $\xi$ :

$$
-(-\nabla)^{\beta / 2} \psi(\xi, \tau)=-\mathscr{F}^{-1}(|\xi| \mathscr{F}(\psi(\xi, \tau))) .
$$

If $\psi$ is defined on $[\mathscr{A}, \mathscr{B}]$ and satisfies $\psi(\mathscr{A}, \tau)=\psi(\mathscr{B}, \tau$ )$=0$, then the function can be extended by taking $\psi(\xi, \tau) \equiv$ 0 for $x \ll a$ and $x \gg b$. Moreover, as shown in [34], if $\psi_{\xi}(\mathscr{A}$ $, \tau)=\psi_{\xi}(\mathscr{B}, \tau)=0$, then the Riesz fractional derivative can be written as follows:

$\frac{\partial^{\beta}}{\partial|\xi|^{\beta}} \psi(\xi, \tau)=-(-\nabla)^{\beta / 2} \psi(\xi, \tau)=-\frac{1}{2 \cos (\pi \beta / 2)}\left[{ }_{a} D_{\xi}^{\beta} \psi(\xi, \tau)+{ }_{\xi} D_{b}^{\beta} \psi(\xi, \tau)\right]$.

The left and right RL-FDs of the Legendre polynomial are given by the following:

$$
\begin{aligned}
& { }_{-1} D_{\xi}^{\mu} P_{k}(\xi)=\sum_{k=0}^{j} \frac{(-1)^{k+j} \Gamma(k+j+1)}{(j-k) ! \Gamma(k+1) 2^{k} \Gamma(k-\mu+1)}(\xi+1)^{k-\mu}, \\
& { }_{\xi} D_{1}^{\mu} P_{k}(\xi)=\sum_{k=0}^{j} \frac{(-1)^{k} \Gamma(k+j+1)}{(j-k) ! \Gamma(k+1) 2^{k} \Gamma(k-\mu+1)}(1-\xi)^{k-\mu} .
\end{aligned}
$$

\section{Chen-Lee-Liu Equation}

In this section, we treat the next nonlinear Riesz space ChenLee-Liu equation

$$
i \frac{\partial \Theta(\xi, \tau)}{\partial \tau}+\frac{\partial^{\mu} \Theta(\xi, \tau)}{\partial|\xi|^{\mu}}+i \gamma|\Theta(\xi, \tau)|^{2} \frac{\partial \Theta(\xi, \tau)}{\partial \xi}=\Delta(\xi, \tau), \quad(\xi, \tau) \in\left[0, \xi_{\text {end }}\right] \times\left[0, \tau_{\text {end }}\right],
$$

with the following conditions:

$$
\begin{gathered}
\Theta\left(0, \tau_{\text {end }}\right)=\chi_{1}(\tau), \Theta\left(\xi_{\text {end }}, \tau\right)=\chi_{2}(\tau), \quad t \in\left[0, \tau_{\text {end }}\right], \\
\Theta(x, 0)=\phi_{1}(x), \quad x \in\left[0, \xi_{\text {end }}\right] .
\end{gathered}
$$

We now split the complex function $\Theta(\xi, \tau)$ into two real functions $\mathscr{U}(\xi, \tau)$ and $\mathscr{V}(\xi, \tau)$ as follows:

$$
\begin{gathered}
\Theta(\xi, \tau)=\mathscr{U}(\xi, \tau)+i \mathscr{V}(\xi, \tau), \Delta(\xi, \tau)=\Delta(\xi, \tau)+i \Delta_{2}(\xi, \tau), \\
\chi_{1}(\tau)=\eta_{1}(\tau)+i \eta_{3}(\tau), \chi_{2}(\tau)=\eta_{2}(\tau)+i \eta_{4}(\tau), \phi_{1}(x)=\varphi_{1}(x)+i \varphi_{2}(x),
\end{gathered}
$$

where $\mathscr{U}(\xi, \tau), \mathscr{V}(\xi, \tau), \Delta_{1}(\xi, \tau), \operatorname{Delta}_{2}(\xi, \tau), \eta_{1}(\tau), \eta_{3}(\tau), \eta$ 
$2(\tau), \eta_{4}(\tau), \varphi_{1}(x)$, and $\varphi_{2}(x)$ are the real functions. Thereafter,

$$
\begin{aligned}
& \frac{\partial \mathscr{U}(\xi, \tau)}{\partial \tau}+\frac{\partial^{\mu} \mathscr{V}(\xi, \tau)}{\partial|\xi|^{\mu}}+\gamma\left(u^{2}(\xi, \tau)+v^{2}(\xi, \tau)\right) \frac{\partial \mathscr{U}(\xi, \tau)}{\partial \xi}=\Delta_{1}(\xi, \tau), \\
& \frac{\partial \mathscr{V}(\xi, \tau)}{\partial \tau}+\frac{\partial^{\mu} \mathscr{U}(\xi, \tau)}{\partial|\xi|^{\mu}}+\gamma\left(u^{2}(\xi, \tau)+v^{2}(\xi, \tau)\right) \frac{\partial \mathscr{V}(\xi, \tau)}{\partial \xi}=\Delta_{2}(\xi, \tau),
\end{aligned}
$$

with the next conditions:

$$
\begin{aligned}
& \mathscr{U}\left(0, \tau_{\text {end }}\right)=\eta_{1}(\tau), \mathscr{U}\left(\xi_{\text {end }}, \tau\right)=\eta_{2}(\tau), \quad t \in\left[0, \tau_{\text {end }}\right], \\
& \mathscr{V}\left(0, \tau_{\text {end }}\right)=\eta_{3}(\tau), \mathscr{V}\left(\xi_{\text {end }}, \tau\right)=\eta_{4}(\tau), \quad t \in\left[0, \tau_{\text {end }}\right] \\
& \mathscr{U}(x, 0)=\eta_{5}(x), \mathscr{V}(x, 0)=\eta_{6}(x), \quad x \in\left[0, \xi_{\text {end }}\right]
\end{aligned}
$$

3.1. Spatial Discretization. The distribution of shifted LegendreGauss-Lobatto nodes in $\left[0, \xi_{\text {end }}\right]$ is the major feature of considering them in our discretization. Here, we list the basic main of implementing our Legendre-Gauss-Lobatto collocation scheme for converting the nonlinear system (Equations (10) and (11)) into temporal ordinary differential system.

The spectral approximation of $\mathscr{P}(\xi, \tau)$ and $\mathscr{Q}(\xi, \tau)$ is given as follows:

$$
\begin{aligned}
& \mathscr{U}_{\mathcal{N}}(\xi, \tau)=\sum_{j=0}^{\mathcal{N}} \varepsilon_{j}(\tau) \mathscr{P}_{\xi_{\text {end }}, j}(x), \\
& \mathscr{V}_{\mathcal{N}}(\xi, \tau)=\sum_{j=0}^{\mathcal{N}} \varepsilon_{j}(\tau) \mathscr{P}_{\xi_{\text {end }}, j}(x),
\end{aligned}
$$

where the orthogonal property and discrete inner product permit the following:

$$
\begin{aligned}
& \varepsilon_{j}(\tau)=\frac{1}{h_{\xi_{\text {end }}, j}} \sum_{i=0}^{\mathcal{N}} P_{j}\left(\xi_{\xi_{\text {end }}, \mathcal{N}, i}\right) \omega_{\xi_{\text {end }}, \mathcal{N}, i} \mathscr{U}\left(\xi_{\xi_{\text {end }}, \mathcal{N}, i} \tau\right), \\
& \varepsilon_{j}(\tau)=\frac{1}{h_{\xi_{\text {end }}, j}} \sum_{i=0}^{\mathcal{N}} P_{j}\left(\xi_{\xi_{\text {end }}, \mathcal{N}, i}\right) \omega_{\xi_{\text {end }}, \mathcal{N}, i} \mathscr{V}\left(\xi_{\xi_{\text {end }}, \mathcal{N}, i} \tau\right) .
\end{aligned}
$$

In that case, Equation (14) takes the form:

$$
\begin{aligned}
& \mathscr{U}(\xi, \tau)=\sum_{i=0}^{\mathcal{N}}\left(\sum_{j=0}^{\mathcal{N}} \frac{1}{h_{\xi_{\text {end }}, j}} \mathscr{P}_{\xi_{\text {end }}, j}\left(\xi_{\xi_{\text {end }}, \mathcal{N}, i}\right) \mathscr{P}_{\xi_{\text {end }, j}, j}(x){\omega_{\xi_{\text {end }}, \mathcal{N}, i}}\right) \mathcal{U}\left(\xi_{\xi_{\text {end },}, \mathcal{N}, i}, \tau\right), \\
& \mathscr{V}(\xi, \tau)=\sum_{i=0}^{\mathcal{N}}\left(\sum_{j=0}^{\mathcal{N}} \frac{1}{\xi_{\xi_{\text {end }}, j}} \mathscr{P}_{\xi_{\text {end }, j}}\left(\xi_{\xi_{\text {end }}, \mathscr{N}, i}\right) \mathscr{P}_{\xi_{\text {end }, j}}(x) \varrho_{\xi_{\text {end }}, \mathcal{N}, i}\right) \mathscr{V}\left(\xi_{\xi_{\text {end },}, \mathcal{N}, i}, \tau\right) .
\end{aligned}
$$

Over and above that, the partial derivative of first order in space evaluated at shifted Legendre-Gauss-Lobatto colloca-
TABLE 1: Maximum absolute errors of Equation (32).

\begin{tabular}{lccc}
\hline$(\mathcal{N}, \mathscr{M})$ & $\mathscr{M}_{\mathcal{U N}_{\mathcal{N}, \mathcal{M}}}$ & $\mathscr{M}_{V \mathcal{N}, \mathscr{M}}$ & $M_{N, M}$ \\
\hline$(2,2)$ & $1.5625 \times 10^{-2}$ & $7.39136 \times 10^{-3}$ & $1.5625 \times 10^{-2}$ \\
$(4,4)$ & $7.01531 \times 10^{-3}$ & $2.43449 \times 10^{-3}$ & $7.01531 \times 10^{-3}$ \\
$(6,6)$ & $1.26263 \times 10^{-3}$ & $4.44263 \times 10^{-4}$ & $1.26263 \times 10^{-3}$ \\
$(8,8)$ & $6.75387 \times 10^{-13}$ & $1.47693 \times 10^{-12}$ & $1.50175 \times 10^{-12}$ \\
$(10,10)$ & $4.35416 \times 10^{-16}$ & $9.29812 \times 10^{-16}$ & $9.56769 \times 10^{-16}$ \\
$(12,12)$ & $5.73001 \times 10^{-17}$ & $2.48174 \times 10^{-16}$ & $2.54703 \times 10^{-16}$ \\
\hline
\end{tabular}

tion is as follows:

$$
\begin{gathered}
\frac{\partial \mathcal{U}\left(\xi_{\xi_{\text {end },}, n}, \tau\right)}{\partial \xi}=\sum_{i=0}^{\mathcal{N}} \rho_{n, i} \mathcal{U}\left(\xi_{\xi_{\text {end }}, \mathcal{N}, i}, \tau\right), \\
\frac{\partial \mathscr{V}\left(\xi_{\xi_{\text {end }}, \mathcal{N}, n}, \tau\right)}{\partial \xi}=\sum_{i=0}^{\mathcal{N}} \rho_{n, i} \mathscr{V}\left(\xi_{\xi_{\text {end }}, \mathcal{N}, i}, \tau\right), \quad n=0,1, \cdots, N,
\end{gathered}
$$

where

$$
\rho_{n, i}=\left.\sum_{j=0}^{N} \frac{\mathscr{\xi}_{\xi_{\text {end }}, \mathcal{N}, i}}{h_{\xi_{\text {end }}, j}} \mathscr{P}_{\xi_{\text {end }}, j}\left(\xi_{\xi_{\text {end }}, \mathcal{N}, i}\right)\left(\frac{\partial \mathscr{P}_{\xi_{\text {end }}, j}(x)}{\partial \xi}\right)\right|_{x=\xi_{\xi_{\text {end }}, \mathcal{N}, n}}
$$

Comparable procedure can be performed to the Riesz fractional derivative $\partial^{\mu} \phi_{\mathcal{N}, \mathscr{M}}(\xi, \eta, \tau) / \partial|\xi|^{\mu}$ for space variable to get

$$
\begin{gathered}
\frac{\partial^{\mu} \mathscr{U}\left(\xi_{\xi_{\text {end }}, \mathcal{N}, n}, \tau\right)}{\partial|\xi|^{\mu}}=\sum_{i=0}^{\mathcal{N}} \lambda_{n, i} \mathscr{U}\left(\xi_{\xi_{\text {end }}, \mathcal{N}, i}, \tau\right), \\
\frac{\partial^{\mu} \mathscr{V}\left(\xi_{\xi_{\text {end }}, \mathcal{N}, n}, \tau\right)}{\partial|\xi|^{\mu}}=\sum_{i=0}^{\mathcal{N}} \lambda_{n, i} \mathscr{V}\left(\xi_{\xi_{\text {end }}, \mathcal{N}, i}, \tau\right), \quad n=0,1, \cdots, N,
\end{gathered}
$$

where

$$
\lambda_{n, i}=\left.\sum_{j=0}^{\mathcal{N}} \frac{\omega_{\xi_{\text {end }}, \mathcal{N}, i}}{h_{\xi_{\text {end }}, j}} \mathscr{P}_{\xi_{\text {end }}, j}\left(\xi_{\xi_{\text {end }}, \mathcal{N}, i}\right)\left(\frac{\partial^{\mu} \mathscr{P}_{\xi_{\text {end }}, j}(x)}{\partial|\xi|^{\mu}}\right)\right|_{x=\xi_{\xi_{\text {end }}, \mathcal{N}, n}} .
$$

Combining the boundary conditions with the abovementioned equations and equalizing the residual of Equation (7) 


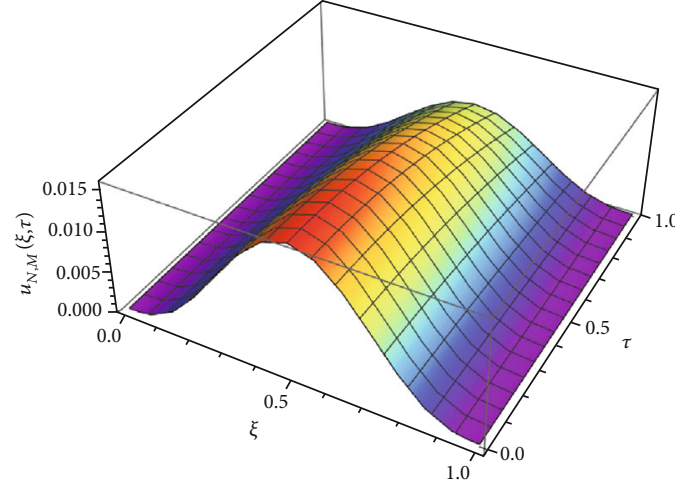

(a) Real part

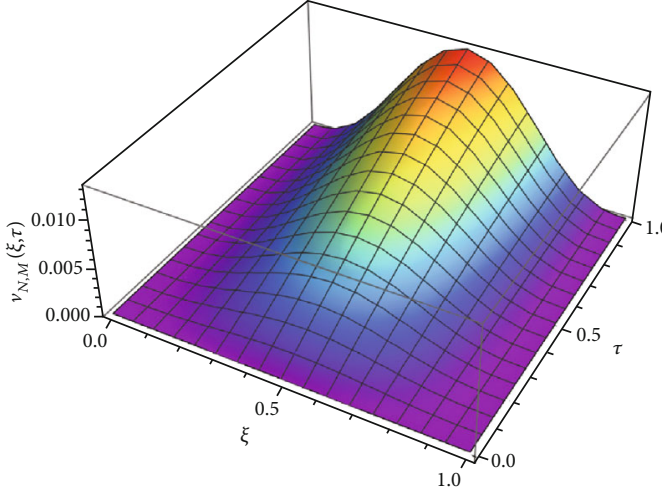

(b) Imaginary part

FIGURE 1: Space graphs of real and imaginary parts of the numerical solution of Equation (32).

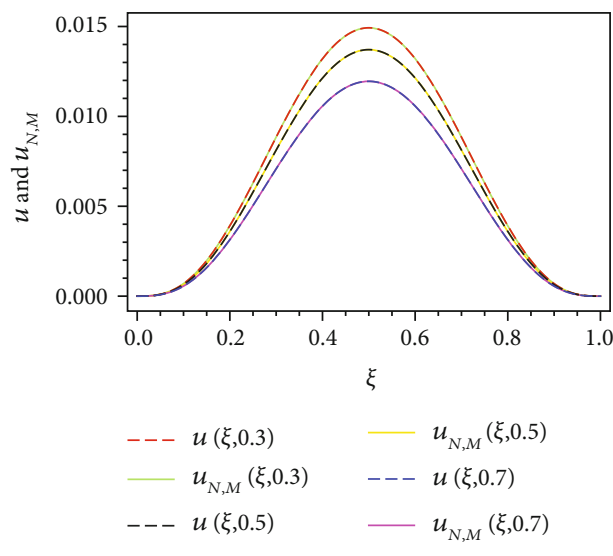

(a) Real part

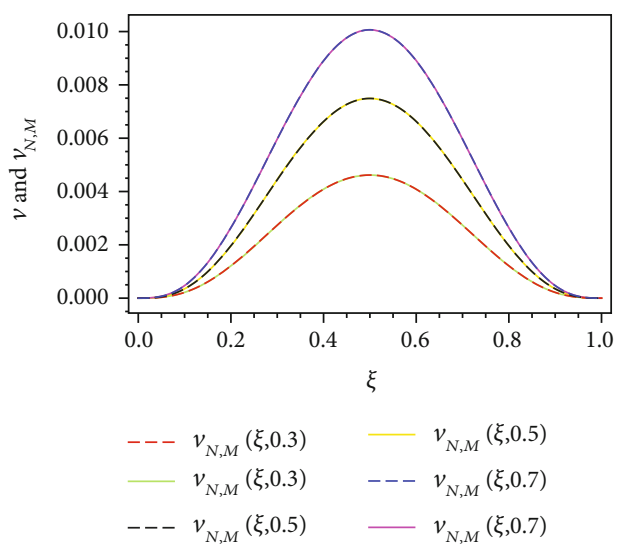

(b) Imaginary part

Figure 2: $\xi$-direction curves for the approximate and exact solutions of real and imaginary parts of Equation (32).

by zero give us the following:

$$
\begin{aligned}
\dot{\mathcal{U}}_{n}(\tau)= & \Delta_{1, n}(\tau)-\sum_{i=1}^{\mathcal{N}-1} \lambda_{n, i} \mathscr{V}_{i}(\tau)-\gamma\left(\mathcal{U}_{n}^{2}(\tau)+\mathscr{V}_{n}^{2}(\tau)\right) \\
& \cdot\left(\rho_{n, 0} \eta_{1}(\tau)+\rho_{n, \mathcal{N}} \eta_{1}(\tau)+\sum_{i=1}^{\mathcal{N}-1} \rho_{n, i} \mathcal{U}_{i}(\tau)\right) \\
& -\lambda_{n, 0} \eta_{3}(\tau)-\lambda_{n, \mathcal{N}} \eta_{4}(\tau), \\
\dot{\mathscr{V}}_{n}(\tau)= & \Delta_{2, n}(\tau)+\sum_{i=1}^{\mathcal{N}-1} \lambda_{n, i} \mathscr{U}_{i}(\tau)-\gamma\left(\mathcal{U}_{n}^{2}(\tau)+\mathscr{V}_{n}^{2}(\tau)\right) \\
& \cdot\left(\rho_{n, 0} \eta_{3}(\tau)+\rho_{n, \mathcal{N}} \eta_{4}(\tau)+\sum_{i=1}^{\mathcal{N}-1} \rho_{n, i} \mathscr{V}_{i}(\tau)\right) \\
& +\lambda_{n, 0} \eta_{1}(\tau)-\lambda_{n, \mathcal{N}} \eta_{2}(\tau), \quad n=1,2, \cdots, \mathcal{N}-1,
\end{aligned}
$$

with initial values

$$
\mathcal{U}_{n}(0)=\eta_{5}(0), \mathscr{V}_{n}(0)=\eta_{6}(0), \quad n=1, \cdots, N-1
$$

where

$$
\begin{aligned}
\mathscr{U}_{k}(\tau) & =\mathscr{U}\left(\xi_{\xi \text { end }, \mathcal{N}, k}, \tau\right), \mathscr{V}_{k}(\tau)=\mathscr{V}\left(\xi_{\xi \text { end }, \mathcal{N}, k}, \tau\right), \Delta_{r, n} \\
& =\Delta_{r}\left(\xi_{\xi \text { end }, N, k}, \tau\right), \quad k=1, \cdots, \mathcal{N}-1, r=1,2 .
\end{aligned}
$$

The numerical approach of such system will be listed in Subsection 3.2.

3.2. Temporal Discretization. Here, we numerically treat the temporal differential system with initial conditions:

$$
\begin{gathered}
\dot{\mathscr{W}}_{r}(\tau)=\mathscr{G}_{r}\left(t, \mathscr{W}_{1}(\tau), \cdots, \mathscr{W}_{\mathscr{R}}(\tau)\right), 0<\alpha<1, \quad r=1, \cdots, \mathscr{R}, t \in\left[0, \tau_{\text {end }}\right], \\
\mathscr{W}_{r}(0)=\tau_{r}, \quad r=1, \cdots, \mathscr{R},
\end{gathered}
$$

where $\mathscr{G}_{r}\left(t, \mathscr{W}_{1}(\tau), \cdots, \mathscr{W}_{\mathscr{R}}(\tau)\right), r=1, \cdots, \mathscr{R}$ are given functions. Shifted Legendre-Gauss-Radau collocation is assigned for temporal discretization, which is more reliable for 


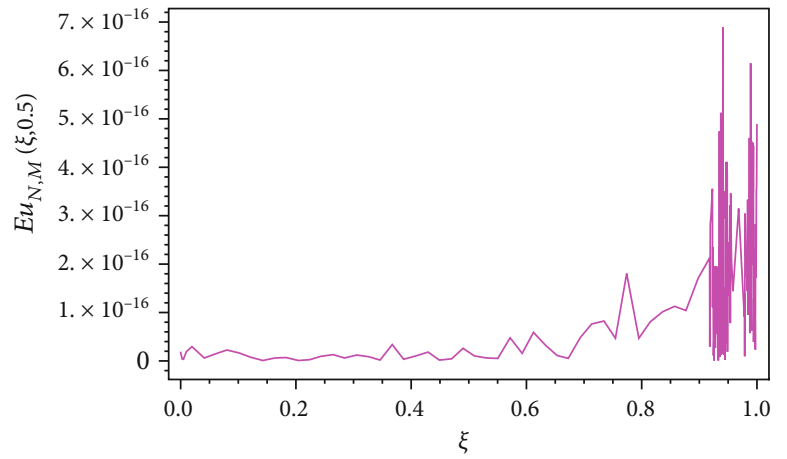

(a) Real part

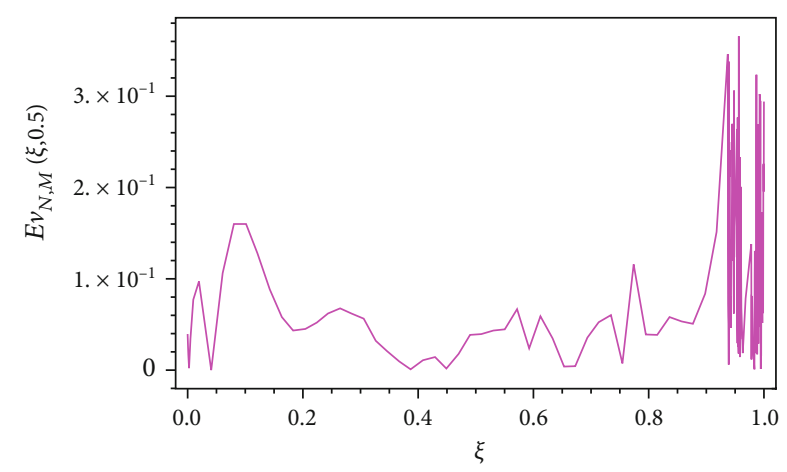

(b) Imaginary part

FIgURE 3: $\xi$-direction curves of real and imaginary parts of the absolute error of Equation (32).

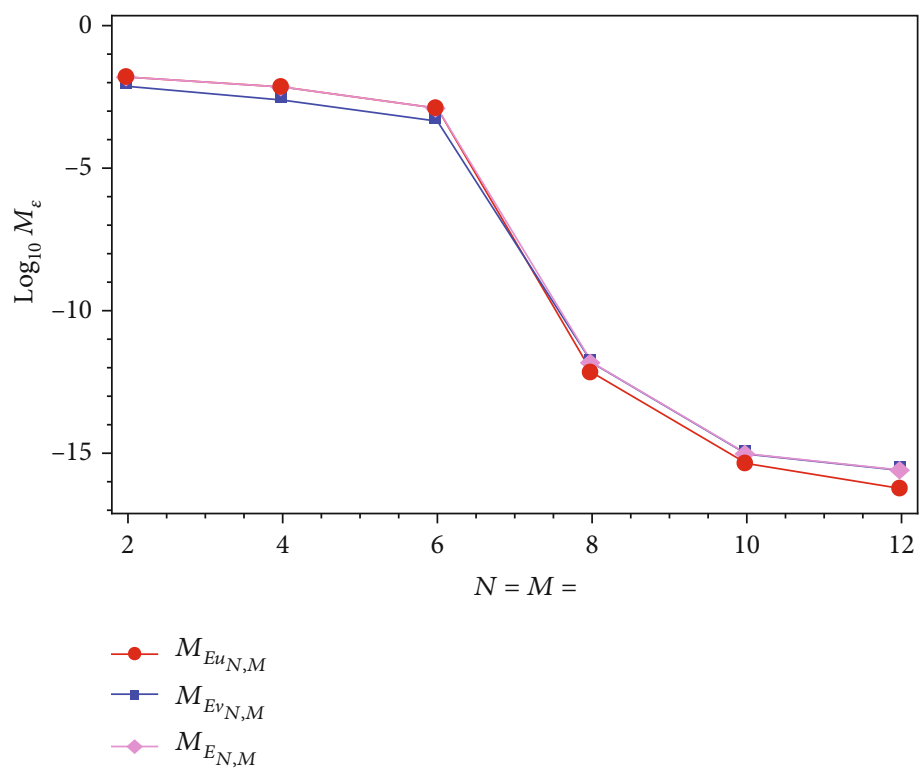

Figure 4: $M_{E}$ convergence of Equation (32).

TABle 2: Maximum absolute errors of Equation (35).

\begin{tabular}{lccc}
\hline$(\mathcal{N}, \mathscr{M})$ & $\mathscr{M}_{U \mathcal{N}, \mathscr{M}}$ & $\mathscr{M}_{V \mathcal{N}, \mathscr{M}}$ & $M_{N, M}$ \\
\hline$(2,2)$ & $3.90625 \times 10^{-3}$ & $3.67244 \times 10^{-3}$ & $3.90625 \times 10^{-3}$ \\
$(4,4)$ & $2.11826 \times 10^{-3}$ & $1.98249 \times 10^{-3}$ & $2.11826 \times 10^{-3}$ \\
$(6,6)$ & $6.60009 \times 10^{-4}$ & $6.04953 \times 10^{-4}$ & $6.60009 \times 10^{-4}$ \\
$(8,8)$ & $8.74126 \times 10^{-5}$ & $7.91024 \times 10^{-5}$ & $8.74126 \times 10^{-5}$ \\
$(10,10)$ & $3.1572 \times 10^{-16}$ & $2.1453 \times 10^{-16}$ & $3.53179 \times 10^{-16}$ \\
$(12,12)$ & $4.75375 \times 10^{-16}$ & $2.13208 \times 10^{-16}$ & $4.86791 \times 10^{-16}$ \\
\hline
\end{tabular}

treating with such problems. We approximate $\mathscr{W}_{r}(\tau)$ as follows:

$$
\mathscr{W}_{r}(\tau)=\sum_{j=0}^{\mathscr{K}} a_{r, j} \mathscr{C}_{\tau_{\text {end }}, j}(\tau), \quad r=1, \cdots, \mathscr{R}
$$

The temporal derivative $\dot{\mathscr{W}}_{r}(\tau)$ is evaluated as follows:

$$
\dot{\mathscr{W}}_{r}(\tau)=\sum_{j=0}^{\mathscr{K}} a_{r, j} \frac{d}{d \tau}\left(\mathscr{C}_{\tau_{\text {end }}, j}(\tau)\right)=\sum_{j=0}^{\mathscr{K}} a_{r, j} \mathscr{C}_{\tau_{\text {end }}, j}^{(1)}(\tau), \quad r=1, \cdots, \mathscr{R} .
$$

Thus, we get the following:

$$
\begin{aligned}
\sum_{j=0}^{\mathscr{K}} a_{r, j} \mathscr{C}_{\tau_{\text {end }}, j}^{(1)}(\tau)= & \mathscr{G}_{r}\left(t, \sum_{j=0}^{\mathscr{K}} a_{1, j} \mathscr{C}_{\tau_{\text {end }}, j}(\tau), \cdots, \sum_{j=0}^{\mathscr{K}} a_{\mathscr{K}, j} \mathscr{C}_{\tau_{\text {end }}, j}(\tau)\right), \quad r=1, \\
& \ldots, \mathscr{R}, t \in\left[0, \tau_{\text {end }}\right],
\end{aligned}
$$

$$
\sum_{j=0}^{\mathscr{K}} a_{r, j} \mathscr{C}_{\tau_{\text {end }, j}}(0)=\tau_{r}, \quad r=1, \cdots, \mathscr{R} .
$$

Combining the initial conditions with the abovementioned equations and equalizing the residual of Equation 


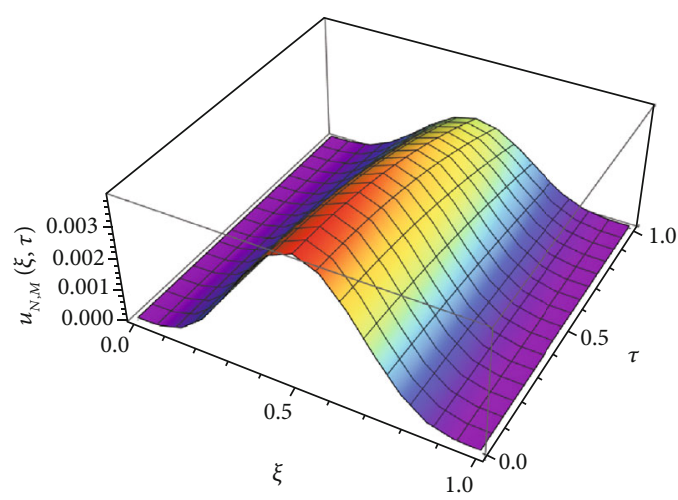

(a) Real part

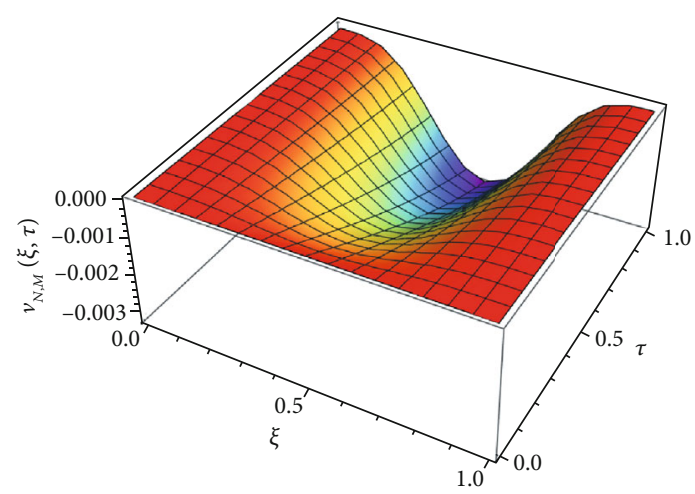

(b) Imaginary part

FIgURE 5: Space graphs of real and imaginary parts of the numerical solution of Equation (35).

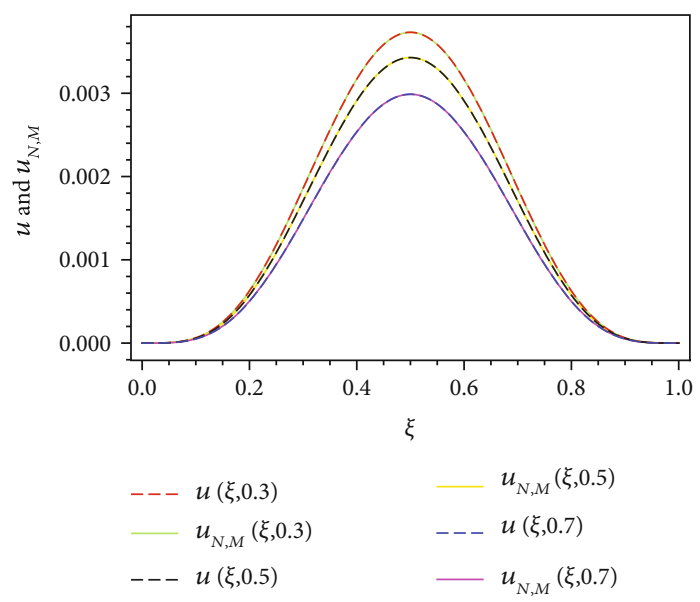

(a) Real part

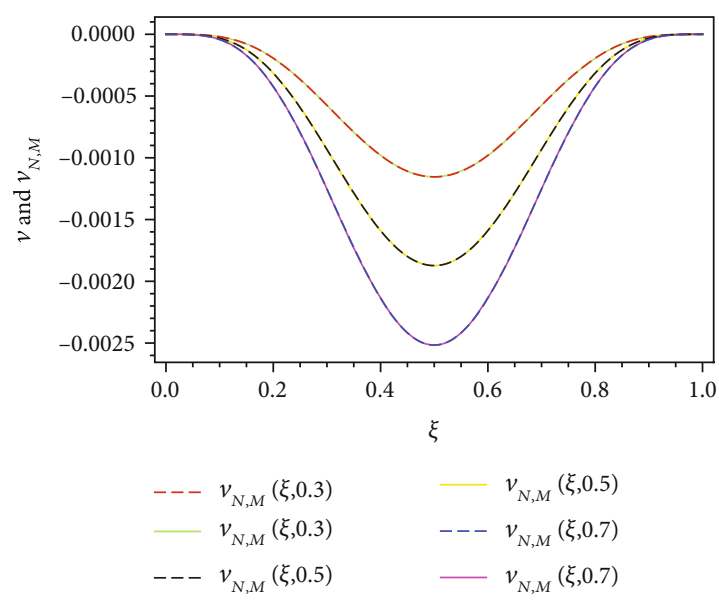

(b) Imaginary part

FIGURE 6: $\xi$-direction curves for the approximate and exact solutions of real and imaginary parts of Equation (35).

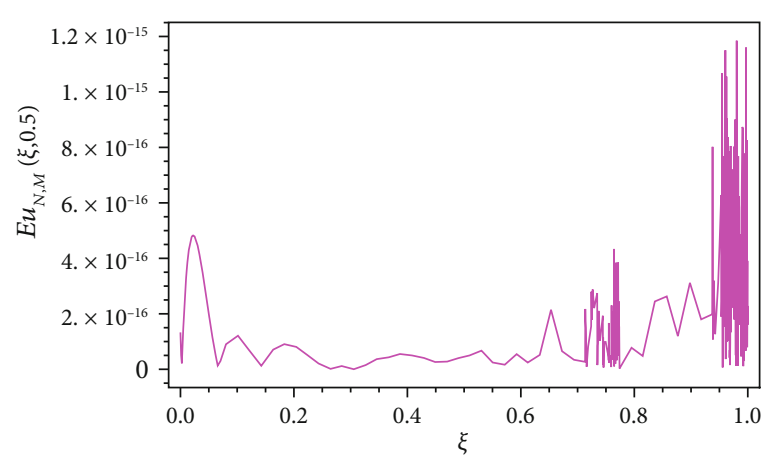

(a) Real part

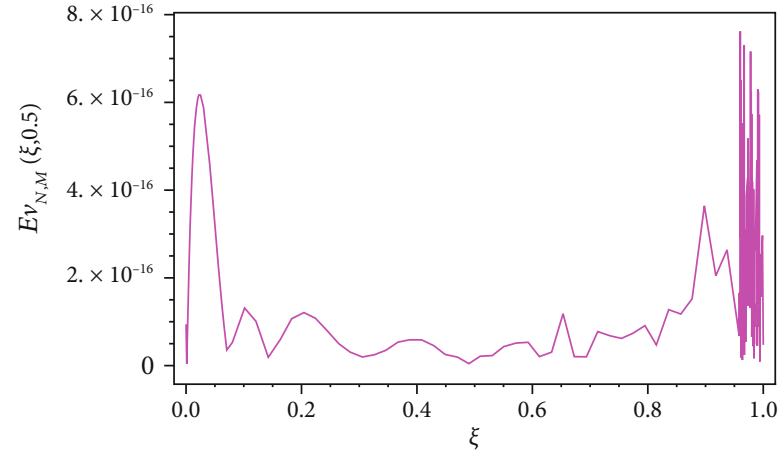

(b) Imaginary part

FIgURE 7: $\xi$-direction curves of real and imaginary parts of the absolute error of Equation (35).

(24) by zero at $(\mathscr{R} \mathscr{K})$ shifted Legendre-Gauss-Radau collocation points give us the following:

$$
\begin{aligned}
\sum_{j=0}^{\mathscr{K}} a_{r, j} \mathscr{C}_{\tau_{\text {end }}, j}^{(1)}= & \mathscr{G}_{r}\left(t, \sum_{j=0}^{\mathscr{K}} a_{1, j} \mathscr{C}_{\tau_{\text {end } j} j}\left(\tau_{\tau_{\text {end }}} \mathscr{K}, s\right), \cdots, \sum_{j=0}^{\mathscr{K}} a_{\mathscr{K}, j} \mathscr{C}_{\tau_{\text {end } j} j}\left(\tau_{\tau_{\text {end }}}, \mathscr{K}, s\right)\right), \quad r=1, \\
& \ldots, \mathscr{R}, s=1, \cdots, \mathscr{K},
\end{aligned}
$$

where the rest $(\mathscr{R})$ algebraic equations are outputted by the initial conditions as follows:

$$
\sum_{j=0}^{\mathscr{K}} a_{r, j} \mathscr{C}_{\tau_{\text {end }}, j}(0)=\tau_{r}, \quad r=1, \cdots, \mathscr{R} .
$$



lows:

Finally, we have $(\mathscr{R}(\mathscr{K}+1))$ algebraic equations as fol-

$$
\begin{aligned}
& \sum_{j=0}^{\mathscr{K}} a_{r, j} \mathscr{C}_{\tau_{\text {end } j}(1)}^{\left(\tau_{\tau_{\text {end }}}, \mathscr{K}, s\right)} \\
& =\mathscr{G}_{r}\left(t, \sum_{j=0}^{\mathscr{K}} a_{1, j} \mathscr{C}_{\tau_{\text {end } j}, j}\left(\tau_{\tau_{\text {end }}}, \mathscr{K}, s\right), \cdots, \sum_{j=0}^{\mathscr{K}} a_{\mathscr{K}, j} \mathscr{C}_{\tau_{\text {end } j}, j}\left(\tau_{\tau_{\text {end }}}, \mathscr{K}, s\right)\right), \quad r=1, \\
& \quad \cdots, \mathscr{R}, s=1, \cdots, \mathscr{K}, \\
& \sum_{j=0}^{\mathscr{K}} a_{r, j} \mathscr{C}_{\tau_{\text {end }}, j}(0)=\tau_{r}, \quad r=1, \cdots, \mathscr{R} .
\end{aligned}
$$

The numerical approach of the previous system will be acquired by using Newton's iterative method.

\section{Applications and Numerical Results}

Here, the adequacy of the spectral collocation algorithms is verified by the obtained results. Problems including initialboundary conditions are examined. Mathematica version 10 is utilized to carry out the code.

Example 1. We test the next problem:

$$
i \frac{\partial \Theta}{\partial \tau}+\frac{\partial^{\mu} \Theta}{\partial|\xi|^{\mu}}+i|\Theta|^{2} \frac{\partial \Theta}{\partial \tau}=\Delta(\xi, \tau),(\xi, \tau) \in[0,1] \times[0,1]
$$

where the function $\Delta(\xi, \tau)$, initial condition, and the boundary conditions are given such as the continuous problem has the next exact solution:

$$
\Theta(\xi, \tau)=e^{i \tau} \xi^{3}(1-\xi)^{3} .
$$

In Table 1, the numerical results based on the maximum absolute errors of Equation (32) obtained using the previous algorithms are listed, where

$$
\begin{gathered}
E_{\mathscr{U}_{\mathcal{N}, M}}(\xi, \tau)=\left|\mathscr{U}_{\mathcal{N}, M}(\xi, \tau)-\mathscr{U}(\xi, \tau)\right|,(\xi, \tau) \in\left[0, \xi_{\mathrm{end}}\right], \\
E_{\mathscr{V}_{\mathcal{N}, M}}(\xi, \tau)=\left|\mathscr{V}_{\mathcal{N}, M}(\xi, \tau)-\mathscr{V}(\xi, \tau)\right|,(\xi, \tau) \in[0, \xi], \\
E_{\mathscr{V}_{\mathcal{N}, M}}(\xi, \tau)=\sqrt{\left(E_{\mathcal{U}_{\mathcal{N}, M}}(\xi, \tau)\right)^{2}+\left(E_{\mathscr{V}_{\mathcal{N}, M}}(\xi, \tau)\right)^{2}},(\xi, \tau) \in[0, \xi], \\
M_{\mathcal{U}_{\mathcal{N}, M}}(\xi, \tau)=\operatorname{Max}\left\{E_{\mathcal{U}_{\mathcal{N}, M}}(\xi, \tau), \quad \forall(\xi, \tau) \in[0, \xi]\right\}, \\
M_{\mathscr{V}_{\mathcal{N}, M}}(\xi, \tau)=\operatorname{Max}\left\{E_{\mathscr{V}_{\mathcal{N}, M}}(\xi, \tau), \quad \forall(\xi, \tau) \in[0, \xi]\right\}, \\
M_{\mathcal{N}, M}(\xi, \tau)=\operatorname{Max}\left\{E_{\mathcal{N}, M}(\xi, \tau), \quad \forall(\xi, \tau) \in[0, \xi]\right\} .
\end{gathered}
$$

Space graphs of real and imaginary parts of the numerical solution of Equation (32) are shown in Figures 1(a) and 1(b), respectively, where $\mathcal{N}=\mathscr{M}=12$. While in Figures 2(a) and 2(b), we recognize the outright matching of numerical and exact solutions in its real and imaginary parts of Equation (32), where $N=M=12$. Also, $\xi$-direction curves for real and imaginary parts of the absolute errors of Equation (32) are plotted in Figures 3(a) and 3(b), respectively, where $\tau=$ $0.5, \mathcal{N}=\mathscr{M}=12$. Moreover, we sketched in Figure 4 the logarithmic graphs of $M_{\varepsilon}$ (i.e., $\log _{10} M_{\varepsilon}$ ) of Equation (32) obtained by the present method with different values of $(\mathcal{N}$ $=\mathscr{M}=2,4,6, \cdots, 12)$.

Example 2. Now, consider the following:

$$
i \frac{\partial \Theta}{\partial \tau}+\frac{\partial^{\mu} \Theta}{\partial|\xi|^{\mu}}+i|\Theta|^{2} \frac{\partial \Theta}{\partial \tau}=\Delta(\xi, \tau), \quad(\xi, \tau) \in[0,1] \times[0,1],
$$

where the function $\Delta(\xi, \tau)$, initial condition, and the boundary conditions are given such as the continuous problem has the next exact solution:

$$
\Theta(\xi, \tau)=e^{i \tau} \xi^{4}(1-\xi)^{4}
$$

In Table 2, the numerical results based on the maximum absolute errors of Equation (35) obtained using the previous algorithms are listed. Space graphs of real and imaginary parts of the numerical solution of Equation (35) are shown in Figures 5(a) and 5(b), respectively, where $\mathcal{N}=\mathscr{M}=12$. While in Figures 6(a) and 6(b), we recognize the outright matching of numerical and exact solutions in its real and imaginary parts, respectively, where $\mathcal{N}=\mathscr{M}=12$. Also, $\xi$ -direction curves for real and imaginary parts of absolute errors of Equation (35) are plotted in Figures 7(a) and 7(b), respectively, where $\tau=0.5, \mathcal{N}=\mathscr{M}=12$. Even though few values of $N$ and $M$, the accurate results have been spotted in these tables. This is consistent with which was predicted in case of using a spectral collocation method. Likewise, these results bring to light the responsibility convergence of the shifted Legendre collocation method for such problems.

\section{Conclusions}

This paper adopted fully collocation method to study Riesz fractional Chen-Lee-Liu equation that discusses soliton propagation down the optical fibers with perturbation terms incorporated into the waveguides. The powerful numerical scheme gave way to a number of impressive numerical results that prove high efficiency of the algorithm. The study was carried out with initial-boundary conditions.

The results of the algorithm pave way to conduct further additional research in this field to display additional results in future. One avenue is to consider Riesz fractional Chen-LeeLiu equation with differential group delay and then further along study the model with additional optoelectronic devices such as in magneto-optic waveguides. Subsequently, this model will be treated with the same algorithm for dense wavelength division multiplexing (DWDM) topology.

Thus, a lot lies in the bucket list!

\section{Data Availability}

There is no data used for this research. 


\section{Conflicts of Interest}

The authors declare that they have no conflicts of interest.

\section{References}

[1] F. S. Sousa, C. F. Lages, J. L. Ansoni, A. Castelo, and A. Simao, "A finite difference method with meshless interpolation for incompressible flows in non-graded tree-based grids," Journal of Computational Physics, vol. 396, pp. 848-866, 2019.

[2] N. A. Mbroh and J. B. Munyakazi, "A fitted operator finite difference method of lines for singularly perturbed parabolic convection-diffusion problems," Mathematics and Computers in Simulation, vol. 165, pp. 156-171, 2019.

[3] H. M. Patil and R. Maniyeri, "Finite difference method based analysis of bio-heat transfer in human breast cyst," Thermal Science and Engineering Progress, vol. 10, pp. 42-47, 2019.

[4] P.-W. Li, F. Zhuo-Jia, G. Yan, and L. Song, "The generalized finite difference method for the inverse Cauchy problem in two-dimensional isotropic linear elasticity," International Journal of Solids and Structures, vol. 174, pp. 69-84, 2019.

[5] S. Yu, J. Li, and C. Zhang, "A local and parallel Uzawa finite element method for the generalized Navier-Stokes equations," Applied Mathematics and Computation, vol. 387, p. 124671, 2020.

[6] C. Wang and J. Wang, "Primal-dual weak Galerkin finite element methods for elliptic Cauchy problems," Computers \& Mathematics with Applications, vol. 79, no. 3, pp. 746-763, 2020.

[7] X. Xiao, Z. Dai, and X. Feng, "A positivity preserving characteristic finite element method for solving the transport and convection-diffusion-reaction equations on general surfaces," Computer Physics Communications, vol. 247, p. 106941, 2020.

[8] E. H. Doha, M. A. Abdelkawy, A. Z. Amin, and A. M. Lopes, "Shifted Jacobi-Gauss-collocation with convergence analysis for fractional integro-differential equations," Communications in Nonlinear Science and Numerical Simulation, vol. 72, pp. 342-359, 2019.

[9] E. H. Doha, A. H. Bhrawy, and S. S. Ezz-Eldien, "A Chebyshev spectral method based on operational matrix for initial and boundary value problems of fractional order," Computers \& Mathematics with Applications, vol. 62, no. 5, pp. 2364-2373, 2011.

[10] H. Ali, "A review of operational matrices and spectral techniques for fractional calculus," Nonlinear Dynamics, vol. 81, no. 3, pp. 1023-1052, 2015.

[11] A. H. Bhrawy and M. A. Zaky, "A method based on the Jacobi tau approximation for solving multi-term time-space fractional partial differential equations," Journal of Computational Physics, vol. 281, pp. 876-895, 2015.

[12] R. M. Hafez and Y. H. Youssri, "Jacobi collocation scheme for variable-order fractional reaction-subdiffusion equation," Computational and Applied Mathematics, vol. 37, no. 4, pp. 5315-5333, 2018.

[13] R. M. Hafez and Y. H. Youssri, "Shifted Jacobi collocation scheme for multidimensional time-fractional order telegraph equation," Iranian Journal of Numerical Analysis and Optimization, vol. 10, no. 1, pp. 195-223, 2020.

[14] A. H. Bhrawy and M. A. Abdelkawy, "A fully spectral collocation approximation for multi-dimensional fractional Schrodinger equations," Journal of Computational Physics, vol. 294, pp. 462-483, 2015.
[15] A. H. Bhrawy, E. H. Doha, D. Baleanu, and S. S. Ezz-Eldien, "A spectral tau algorithm based on Jacobi operational matrix for numerical solution of time fractional diffusion-wave equations," Journal of Computational Physics, vol. 293, pp. 142156, 2015.

[16] R. M. Hafez, M. A. Zaky, and M. A. Abdelkawy, "Jacobi spectral Galerkin method for distributed-order fractional RayleighStokes problem for a generalized second grade fluid," Frontiers in Physics, vol. 7, 2020.

[17] E. H. Doha and W. M. Abd-Elhameed, "Efficient spectral ultraspherical-dual-Petrov-Galerkin algorithms for the direct solution of $(2 \mathrm{n}+1)$ th-order linear differential equations," Mathematics and Computers in Simulation, vol. 79, no. 11, pp. 3221-3242, 2009.

[18] A.-M. Wazwaz and L. Kaur, "Optical solitons and peregrine solitons for nonlinear Schrödinger equation by variational iteration method,” Optik, vol. 179, pp. 804-809, 2019.

[19] H. Triki and A.-M. Wazwaz, "Combined optical solitary waves of the Fokas-Lenells equation," Waves in Random and Complex Media, vol. 27, no. 4, pp. 587-593, 2017.

[20] A.-M. Wazwaz and L. Kaur, "Complex simplified Hirota's forms and Lie symmetry analysis for multiple real and complex soliton solutions of the modified KdV-Sine-Gordon equation," Nonlinear Dynamics, vol. 95, no. 3, pp. 2209$2215,2019$.

[21] A.-M. Wazwaz, "Two-mode fifth-order KdV equations: necessary conditions for multiple-soliton solutions to exist," Nonlinear Dynamics, vol. 87, no. 3, pp. 1685-1691, 2017.

[22] E. H. Doha, A. H. Bhrawy, M. A. Abdelkawy, and R. A. Van Gorder, "Jacobi-Gauss-Lobatto collocation method for the numerical solution of $1+1$ nonlinear Schrödinger equations," Journal of Computational Physics, vol. 261, pp. 244-255, 2014.

[23] H. Wang, "Numerical studies on the split-step finite difference method for nonlinear Schrödinger equations," Applied Mathematics and Computation, vol. 170, no. 1, pp. 17-35, 2005.

[24] M. Dehghan and A. Taleei, "Numerical solution of nonlinear Schrödinger equation by using time-space pseudo-spectral method," Numerical Methods for Partial Differential Equations: An International Journal, vol. 26, no. 4, pp. 979-992, 2010.

[25] M. Dehghan and A. Taleei, "A Chebyshev pseudospectral multi-domain method for the soliton solution of coupled nonlinear Schrödinger equations," Computer Physics Communications, vol. 182, no. 12, pp. 2519-2529, 2011.

[26] L. Kaur and A.-M. Wazwaz, "Bright-dark optical solitons for Schrödinger-Hirota equation with variable coefficients," Optik, vol. 179, pp. 479-484, 2019.

[27] H. H. Chen, Y. C. Lee, and C. S. Liu, "Integrability of nonlinear Hamiltonian systems by inverse scattering method," Physica Scripta, vol. 20, no. 3-4, p. 490, 1979.

[28] E. Fan, "Integrable systems of derivative nonlinear Schrödinger type and their multi-Hamiltonian structure," Journal of Physics A: Mathematical and General, vol. 34, no. 3, p. 513, 2001.

[29] J. Zhang, W. Liu, D. Qiu, Y. Zhang, K. Porsezian, and J. He, "Rogue wave solutions of a higher-order Chen-Lee-Liu equation," Physica Scripta, vol. 90, no. 5, article 055207, 2015.

[30] A. J.'a. M. Jawad, A. Biswas, Q. Zhou, M. Alfiras, S. P. Moshokoa, and M. Belic, "Chirped singular and combo optical solitons for Chen-Lee-Liu equation with three forms of integration architecture," Optik, vol. 178, pp. 172-177, 2019. 
[31] A. Biswas, M. Ekici, A. Sonmezoglu et al., "Chirped optical solitons of Chen-Lee-Liu equation by extended trial equation scheme," Optik, vol. 156, pp. 999-1006, 2018.

[32] Y. Yıldırım, "Optical solitons to Chen-Lee-Liu model with modified simple equation approach," Optik, vol. 183, pp. 792-796, 2019.

[33] K. S. Miller and B. Ross, An Introduction to the Fractional Calculus and Fractional Differential Equations, Wiley, 1993.

[34] P. Zhuang, F. Liu, V. Anh, and I. Turner, "Numerical methods for the variable-order fractional advection-diffusion equation with a nonlinear source term," SIAM Journal on Numerical Analysis, vol. 47, no. 3, pp. 1760-1781, 2009. 